\title{
A narrative review of targeted therapies in meningioma
}

\section{Lyndon Kim}

Division of Neuro-Oncology, Department of Neurology, Medicine and Neurosurgery, Icahn School of Medicine at Mount Sinai, New York, NY, USA Correspondence to: Lyndon Kim, MD. Mount Sinai Hospital, Icahn School of Medicine at Mount Sinai, 1 Gustave L Levy Pl Box 1137 New York, NY 10029, USA. Email: lyndon.kim@mssm.edu.

\begin{abstract}
Meningiomas are the most common primary brain tumors constituting approximately one third of all primary brain tumors. It affects mainly elderly population with increased incidence older than 65 years of age and more woman than man. It usually follows a benign course with a fairly good outcome and the surgery and or radiation therapy remain the standard of care. The prognosis remains excellent for grade I meningiomas with 10 -year overall survival greater than $90 \%$. However, although the most of the meningiomas, especially for grade I, can be cured by surgery alone, for grades II and III recurrent meningiomas, they become a clinical challenge as there are no clear standard treatment options available after re-resection or re-irradiation therapy. Prognosis is particularly poor for grade III meningiomas with 10 year overall survival of $33 \%$. Many chemotherapeutic agents and hormonal therapies have been tried with only modest benefits. Recent advances in molecular genetic profiling and diagnostic tools greatly enhanced our understanding of the complex pathways and it opened an opportunity for potential targeted therapies for specific markers. Clinical trial results from sunitinib [multitargeted tyrosine kinase inhibitor (TKI)], bevacizumab (VEGF inhibitor), everolimus (mTOR inhibitor) and bevacizumab revealed some promising tumor response in recurrent meningiomas. Currently, many clinical trials including targeted therapies, antiangiogenic agents and immunotherapies are being investigated or under consideration.
\end{abstract}

Keywords: Meningioma; chemotherapy; hormonal therapy; immunotherapy; molecular targeted therapy; bevacizumab; sunitinib; everolimus

Submitted Feb 13, 2020. Accepted for publication Dec 09, 2020.

doi: $10.21037 / \mathrm{cco}-2020$-mbt-01

View this article at: http://dx.doi.org/10.21037/cco-2020-mbt-01

\section{Introduction}

Meningiomas are the most common primary brain tumors constituting approximately $37 \%$ of these cases. They usually affect the elderly population ( 65 years old) and have a female preponderance of approximately 2:1 ratio over male (10.83:4.83 per 100,000 persons per year), however, grades II and III occur more often in males. Incidence of meningiomas is 27,000 per year (male:female $=7,200: 19,800$ cases per year) and the prevalence is 170,000 in the United States $(1,2)$. The tumor is associated with previous ionizing radiation, such as treatment of tinea capitis, atomic bomb survivors and patients who received radiation to the head and neck, and germline mutations in the NF2, SMARCB1, LZTR1 or SMARCE1 genes (3-6). Multiple studies have evaluated the hormonal risk in woman, cellular phone use, diet and allergy history, head traumas and occupational exposures, all without conclusive results.

Meningiomas can be found incidentally without symptoms and they can be simply followed with serial scans for periodic observation. Most cases that result in symptoms can be treated with surgery and radiation therapy can be added depending on the presence of residual tumor and histologic type and grade. Frequent symptoms and signs include headaches, new onset of seizures, mental status changes, focal weakness, speech difficulty and cranial nerve deficits depending on the location of the tumor. Meningiomas appear extra-axial and vascular on computed tomography (CT) and magnetic resonance imaging (MRI) of brain. Calcifications and bone involvement can be seen on CT scan. MRI scan usually reveals a dural based homogeneously enhancing lesion that has a typical tail sign. Calcification usually confers a benign low grade 


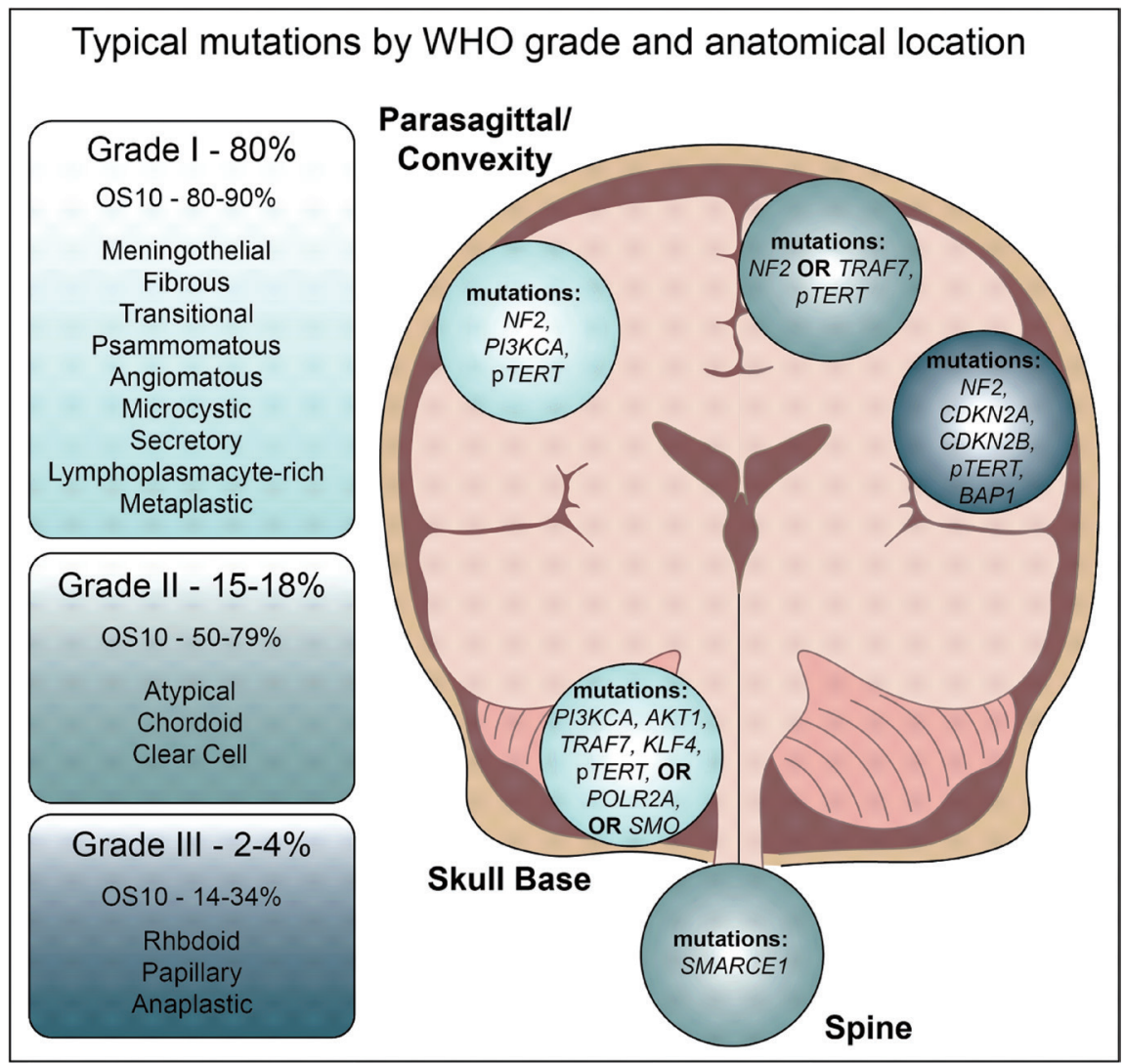

Figure 1 Typical mutations by WHO classification and anatomical location (7-11).

meningiomas while vasogenic edema are often seen in highgrade meningiomas.

Meningiomas arise from the meninges which is composed of the three protective membranes, dura mater, arachnoid mater, and pia mater, that line the skull and vertebral canal and enclose the brain and spinal cord. Meningiomas are graded based on WHO criteria from grade I, II and III which are further divided into 15 subtypes $(7,8)$ (Figure 1) and mostly, they are considered benign compared to more aggressive tumors such as malignant gliomas.

Grade I meningiomas which constitute approximately $80 \%$ are slow growing tumors that can be potentially curable if gross total resection can be achieved. Ten-year overall survival (OS) rate is around $80-90 \%$. The most common subtypes include meningothelial, fibroblastic, and transitional $(8,12)$. Psammomatous, angiomatous, microcytic, secretory, lymphoplasmacyte-rich and metaplasia are less common (8).

Grade II meningiomas are called atypical meningiomas which represent $15-18 \%$ of all meningiomas. It is characterized by the increased mitotic activity, presence of brain invasion, or by the presence of at least three of the following morphological criteria; small cells with a high nuclear to cytoplasmic ratio, high cellularity, sheeting, uninterrupted growth pattern, foci of necrosis, or prominent nucleoli. The tumor has mitotic proliferation index of 4-19 per 10 high powered fields compared to $<4 \%$ in grade I. The tumor is classified as chordoid, clear cell and atypical meningioma (8). This tumor is still considered as benign and most of these tumors can be closely observed after surgery. Radiation therapy can be an option for symptomatic patients, incomplete resection of tumor, presence of high mitotic proliferation index or brain invasion. Current ongoing NRG Oncology BN-003 clinical trial randomizing observation versus radiation therapy will answer how to best treat these patients.

Grade III meningiomas are called malignant meningiomas and they constitute about $2-4 \%$ of all meningiomas. In contrast to lower-grade meningiomas, the incidence is higher in male than female. The prognosis is poor with 
10 -year PFS of $32.8 \%$ (age $>75$ ) compared to $87.1 \%$ of non-malignant meningiomas (age $>40)(13)$. The tumor is characterized by loss of typical architecture, necrosis and brain invasion and they are classified as papillary, rhabdoid and anaplastic $(8,12)$. Their mitotic proliferation index is higher than $20 \%$ and are difficult to treat as they tend to grow rapidly with frequent recurrences, often less than 5 years. Malignant meningioma with anaplastic features such as sarcoma, carcinoma or melanoma like histology is a rapidly growing tumor that carries a poor prognosis with PFS less than 2-3 years. Most of these patients will require additional treatments such as radiation therapy following surgery.

Approximately, 20\%, $40 \%$ and $80 \%$ of WHO grade I, II and III meningiomas, respectively, will recur after the initial surgical resection. About one third of these patients could not have gross total resection at the initial presentation mainly due to its eloquent location and approximately $70 \%$ of these patients will eventually recur.

Recurrent meningiomas can be treated with re-resection if surgery could be an option. If not, these patients can be treated with conventional standard radiation therapy or stereotactic radiosurgery. Most tumors, especially highergrade, will continue to progress with limited additional treatment options. New novel treatments are desperately needed.

I present the following article in accordance with the Narrative Review reporting checklist (available at http:// dx.doi.org/10.21037/cco-2020-mbt-01).

\section{Methods}

A literature search of PubMed was performed to identify publications-including basic science research, clinical studies, and case reports pertaining to meningiomas. No publication limits were applied, and no inclusion or exclusion criteria were implemented for the literature search. The author provides a narrative summary of the findings and implications of these studies, and how they pertain to improving the clinical care of our own patients with these pathologies.

\section{Medical management}

Tamoxifen, mifepristone (RU486), hydroxyurea, alpha interferon and other chemotherapeutic agents have been tried with limited success and relatively high side effects. New innovative treatment options are needed.
Hydroxyurea has shown some modest benefits (14) and may have outcomes equivalent to those using radiation therapy (15). In a phase II clinical trial with cyclophosphamide, adriamycin and vincristine, 3 patients had partial response and 11 patients had stable disease. The median PFS was 5.3 years (16). Other chemotherapeutic agents like temozolomide, the standard of therapy for gliomas, were found to have limited activities for the treatment of recurrent or progressive meningiomas (17). However, if the tumor is found to have MGMT methylation, temozolomide could be considered.

\section{Targeted therapies}

The role of chemotherapy and targeted therapies are not well established, however, recent advances in molecular genetic profiling are revealing unique markers that make it potential targets for treatment.

There was a high anticipation to hormonal therapies such as progesterone and androgen antagonists based on the fact that as much as $65 \%$ to $88 \%$ of meningioma patients harbor these receptors with initial response rate of $25 \%$ to mifepristone, a potent progesterone antagonist, in one study and 5/14 (36\%) in another study. However, in a larger phase III trial, this benefit could not be reproducible and maybe, a proper stratification by progesterone receptor expression within the context of a trial may help to clarify the utility of this approach (18).

Somatostatin receptor (SSTR2) is present in about $90 \%$ of meningiomas which makes it potential target for Sandostatin LAR which is a somatostatin agonist. Octreotide scan, single-photon emission computed tomography (SPECT) or gallium 68 Dotatate scans are done prior to treatment to confirm the presence of octreotide expression. Initial study showed $31 \%$ partial response and $44 \%$ progression-free survival (PFS) at 6 months, however, a multicenter phase II study demonstrated no significant response (19).

Pasireotide which has a higher affinity for somatostatin receptors also revealed no significant benefit. Of 34 evaluable patients, $24(71 \%)$ had stable disease as their best response. Twelve/18 (67\%) patients in cohort A with grades II and III achieved stable disease for a median of 4 cycles (range, $2-12$ ), and $13 / 16$ patients $(81 \%)$ in cohort B with grade I achieved stable disease for a median of 7 cycles (range, 2-35). Progression-free survival at 6 months was $17 \%$ in cohort A and $50 \%$ in cohort B. Although the inhibition of SSTR2 failed to improve the clinical 
outcomes, expression levels of SSTR3 seemed to correlate with increased PFS, warranting further investigation (20).

Interestingly, a recent in vitro analysis of 81 patient derived meningioma cell lines showed a significant antiproliferative effect of octreotide, without apoptotic response, which supports the clinical observation of stable disease without tumor reduction in these trials (21).

Peptide receptor radionuclide therapy (PRRT) may be beneficial for somatostatin receptor positive type IIA (SSTR2A) which is a highly specific marker for meningioma cells. Positron emission tomography (PET) scan with SSTR ligands like gallium 68 Dotatate scan is performed prior to PRRT. This and other somatostatin receptor analogs are used to direct radionuclides for uptake by tumor cells, leading to death in a targeted fashion. Currently, PRRT such as Copper-64 labeled SARTATE, and ${ }^{177}$ Lu-DOTATyr3-octreotate (Lutathera), are in clinical trials for the treatment of meningiomas.

Elevated levels of cancer-associated tyrosine kinase pathways are found in meningioma cells, which are driven by the overexpression of receptors and or their cognate ligands, making them potential targets for tyrosine kinase inhibitors.

\section{Single specific targeted therapies}

EGFR inhibitors with either erlotinib or gefitinib were tested in a phase II recurrent meningioma trial and 8 out of 25 patients showed stable disease but the rest had tumor progression with no significant differences in PFS or PFS (22).

Imatinib, a platelet-derived growth factor receptor (PDGFR) inhibitor, was tested in a phase II clinical trial for recurrent grade I, II and III meningiomas. There were no statistically significant changes seen in PFS or OS. Of 19 patients evaluable for response, 10 patients progressed at the first scan, and 9 patients had stable disease. No complete or partial responses were seen. Overall median PFS was 2 months and PFS at 6 months was 29.4\%. For benign meningiomas (grade I), median PFS was 3 months and PFS at 6 months was $45 \%$. For atypical (grade II) and malignant (grade III) meningiomas, median progression survival was 2 months and PFS at 6 months was $0 \%$. It was felt that it had no significant activity in recurrent meningiomas (23).

The NF2 gene product, Merlin, was found to regulate mTOR signaling negatively. Hence, agents targeting mTOR have been extensively studied for the treatment of NF2 mutated meningiomas. Everolimus, an mTORC1 inhibitor, was investigated in the vestibular schwannoma trial with no radiographic evidence of response (24). Currently, an everolimus trial for recurrent meningiomas is underway.

Vistusertib is a dual mTORC1/C2 Inhibitor which revealed promising preclinical data in blocking proliferation of meningioma cells. A trial for recurrent grade II and III meningiomas and a second trial for NF2 patients with progressive or symptomatic meningiomas are being investigated.

\section{Multi-targeted therapies/combination therapies}

Contrary to the previous single target therapies, sunitinib is a small molecular multi-tyrosine kinase inhibitor that targets VEGFR, PDGFR, FMS-like tyrosine kinase, tyrosine protein kinase KIT, macrophage colonystimulating factor-1 receptor (CSF1R), and RET protooncogene, most of which are abundantly expressed in meningiomas. It has antiangiogenic, cytostatic and antimigratory activity for meningioma cells. A prospective multicenter phase II trial for recurrent and progressive atypical and anaplastic meningiomas was conducted. Thirtysix patients (30 atypical, 6 anaplastic) who were heavily pretreated (median: 5 recurrences) PFS at 6 months was $42 \%$. PFS was 5.2 months and mean PFS was 24.6 months. VEGFR2 expression predicted PFS of 1.4 and 6.4 months in VEGFR2-negative and VEGFR2-positive patients respectively. Responses were mainly stable disease but two had a significant reduction in tumor size. Authors concluded that sunitinib is active in recurrent atypical and anaplastic meningiomas and a randomized trial should be conducted (25).

Antiangiogenic agent, bevacizumab, a VEGF inhibitor, for grades II and III meningiomas revealed 6 months PFS of $43.8 \%$. In addition, a review of 22 studies for a total of 92 patients revealed PFS of 16.8 months with 6 months PFS of $73 \%$. A phase II trial for all grades (I, II and III) recurrent meningiomas, 40 patients were treated: 15 benign meningiomas (grade I), 22 atypical meningiomas (grade II), and 13 malignant meningiomas (grade III). Best responses were stable disease (grade I: $100 \%$, grade II: $85 \%$, grade III: $82 \%)$. Partial response was seen in grade I: $0 \%$, grade II: $5 \%$ and grade III: $0 \%$. Progressive disease was seen in grade I $0 \%$, grade II $10 \%$ and grade III $18 \%$. PFS at 6 months, median PFS and median OS were 87\%, 22.5 months, 35.6 months for grade I, $77 \%, 15.3$ months, not reached for grade II, and $46 \%, 3.7$ months, 12.4 months for grade III respectively (26).

With this encouraging and durable response seen as a 
single agent with bevacizumab, a combination approach with other targeted therapies was explored.

$\mathrm{PI} 3 \mathrm{~K} / \mathrm{AKT} / \mathrm{mTOR}$ pathway plays an important role in the growth of meningioma and AKT1 and mTOR mutation has been seen in $9 \%$ and $7 \%$ of meningioma patients respectively. A phase II clinical trial with everolimus, mTOR inhibitor, plus bevacizumab for the treatment of recurrent or progressive meningioma patients revealed a best response of stable disease in $15 / 17$ patients $(88 \%)$, of which 6 patients had a stable disease for $>12$ months. Overall median PFS was 22 months and was greater for patients with grades II and III (22 months) compared to grade I (17.5 months). The median duration of disease stabilization was 10 months (27).

A phase II study combining everolimus and octreotide for the treatment of refractory and progressive meningioma of all grades was performed and it revealed a radiographic response in 4/20 patients at 3 months and an encouraging PFS at 6 months of $58.2 \%$ and PFS at 12 months of $38 \%$ with a median follow-up of 12.3 months. Pre-therapeutic growth rate was decreased more than 50\% in 29/35 tumors (18/20 patients) during the first 3 months and inclusion (I)-to-3 months tumor growth rate was significantly lower than pre-inclusion (Pre-I)-to-I growth rate. Everolimus and octreotide combination approach appears to be active in refractory aggressive and progressive meningiomas which should warrant further evaluation (28).

Blockade of PI3K signaling, like mTOR inhibition, may not induce apoptosis, however, in vitro data from primary meningioma cell lines demonstrated caspase induced cell death via MEK inhibition, and therefore, PI3K inhibitor, alpelisib, in combination with the MEK inhibitor, trametinib might be effective in the treatment of meningioma and it warrants further investigation.

Next-generation sequencing (NGS) revealed over $50 \%$ of meningiomas have NF2 mutation, $8-13 \%$ have AKT1 mutation, a member of the PI3K/AKT/mTOR pathway, and $3-5 \%$ of non-NF2 meningiomas have SMO mutation, a member of the Hedgehog pathway, that seem to be clinically relevant. NF2-mutant meningiomas are typically atypical meningiomas and originate in the cerebrum or cerebellum. Non-NF2 meningiomas are benign slow growing tumors originating from the skull base.

Recently, a case report of a female patient with metastatic meningotheliomatous meningioma involving the brain and the lung who got treated with pan-AKT inhibitor, AZD5363 for AKT1E17K mutation, showed a promising and durable response. Ex vivo cultured meningioma cells revealed sensitivity to the drug as shown by pan-AKT accumulation on immunoblots. The patient has been treated for more than a year with a radiographic response which warrants further investigation in a proper clinical trial setting (29).

There is an ongoing Alliance clinical trial, using vismodegib, a hedgehog pathway targeting agent, for $\mathrm{SMO} /$ PTCH1 mutated progressive/recurrent meningiomas. For NF2 mutant meningiomas, FAK inhibitor (GSK2256098) will be provided. For AKT1 mutated meningioma patients, an AKT1 targeted therapy (Afuresertib) will be provided.

Tazemetostat, a BAP1 inhibitor, is currently being investigated for the treatment of rhabdoid meningioma that often expresses BAP1 mutation. Rhabdoid meningioma is rare and aggressive malignant tumor (grade III) associated with a great tendency for recurrence and poor outcome that seems to correlate with BAP1 loss. Tazemetostat targets increased level of PCR2 complex protein, EZH2, by BAP1 inactivation. Some mutations may drive epigenetic changes, such as inactivation of the SWI/SNF complex, which disrupts its balance PCR2, leading to altered methylation profiles which might lead to disease formation or progression of disease. As such, the hypermethylation of associated genes in higher-grade meningiomas could be the potential target of EZH2 inhibitors such as tazemetostat (30,31).

$C D K N 2 A, C D K N 2 B$ are the key tumor suppressor genes involved in cell cycle control. Alterations of $C D K N 2 A$, $C D K N 2 B$ genes could be potential genetic driver alterations in atypical and anaplastic meningiomas (32). Recurrent genomic alterations such as $C D K N 2 A$ and $C D K N 2 B$ locus loss on the p-arm of chromosome 9 could lead into the malignant progression/transformation to grade III (33). A clinical trial with CDK inhibitor with ribociclib for recurrent high-grade meningiomas is under consideration.

Other potential target for higher-grade meningiomas is telomerase reverse transcriptase (TERT) promoter mutations which revealed a substantially shorter PFS than TERT promoter wild type meningiomas ( 2.7 versus 10.8 years) in one study that had 26 patients with progressive/ higher grade meningiomas (grade I: 3 patients, grade II: 13 patients, grade III: 10 patients) (34). Additional investigation on TERT promotor mutation on PFS in a clinical trial setting is warranted.

\section{Immunotherapies}

Immunotherapy has shown a promising development in recent years with increased understanding of the tumor 
microenvironment and the body immune system. There have been various efforts to utilize immunotherapeutic modalities or combination therapy for the treatment of systemic malignancies including meningiomas.

The antineoplastic effects of the cytokine, IFN- $\alpha$, have been explored in the treatment of various cancers including melanomas, renal cell carcinomas and meningiomas. In addition, IFN- $\alpha$ appears to have an antiangiogenic activity which could be useful in highly vascular tumors like meningiomas. IFN- $\alpha-2 \mathrm{~B}$ is a leukocyte-produced, naturally occurring IFN, with immunomodulatory and antiproliferative activities. A prospective clinical trial for grade I recurrent meningioma with IFN- $\alpha-2 \mathrm{~B}$ revealed $26 / 35$ patients had stable disease after the first 3 cycles and 9 patients had disease progression. PFS at 6 months was $54 \%$ and at 12 months was $31 \%$. Median time to tumor progression was 7 months. The median PFS was 8 months (35).

The recent advance in understanding immune system brought the potential role of PD-1 and PD-L1 targeting antibodies for the treatment of meningiomas. It was found that signs of $\mathrm{T}$ cell exhaustion and immune evasion, which can lead to decreased levels of $\mathrm{PD}-1^{+} \mathrm{T}$ cells, were commonly found in higher-grade meningiomas especially anaplastic (grade III) meningiomas. The expression of PDL1 was also increased in grade III meningiomas and was associated with worse survival outcome.

Recently, a significant response to nivolumab, a PD-1 Inhibitor, was reported on a recurrent and refractory meningioma patient who was noted to have a homozygous deletion of the DNA mismatch repair (MMR) gene, $M S H 2$. Increased tumor mutational burden and presence of tumor neoantigens are associated with dysfunction of MMR mechanisms which may lead to heightened immune response. Elevated tumor mutation burden was found to be associated with MIB-1 proliferative index, anaplastic histology, and chromosomal instability but not with recurrent tumor, previous radiation therapy or radiation induced meningioma. The dramatic response of an MMRdeficient meningioma to immunotherapy suggests that screening of meningioma patients with MMR should be performed to identify a molecularly-defined subtype that would likely respond to immunotherapy (36).

Recently, FDA approved pembrolizumab, a PD-1 inhibitor, for any solid organ tumor patients who harbor MMR deficiency which makes a unique treatment option for those recurrent meningioma patients with MMR deficiency.
In addition to PD-1 inhibitors, PD-L1 inhibitors are also investigated. PD-L1 is frequently found upregulated in grade II and III meningiomas. Avelumab, an anti PD-L1 inhibitor, is found to induce antibody dependent cellular cytotoxicity in vitro studies using meningioma and natural killer cell lines (37). Avelumab with hypofractionated proton therapy clinical trial for recurrent radiation refractory meningiomas is currently underway.

\section{Additional notable targets and potential therapies}

A recent molecular genetic profiling of 115 meningiomas by multiplatform analysis [NGS, fluorescence in situ hybridization (FISH), immunohistochemistry (IHC) and chromogenic in situ hybridization (CISH)] revealed EGFR (93\%, n=44), PTEN, BCRP, MRP1 and MGMT (55\%, $\mathrm{n}=97)$. The most frequent mutation among all grades gene occurred in the NF2 gene at $85 \%$ [11/13]. Recurring SMO and AKT1 were also occasionally detected. PD-L1 was also expressed in $25 \%$ of grade III cases [2/8] but not in grade I or II tumors. PD- $1^{+} \mathrm{T}$ cells were present in $46 \%$ [24/52] of meningiomas. TOP2A and TS expression increased with grade. If predicated on tumor expression, therapeutics directed toward NF2 and TOP2A could be considered for most meningioma patients and other biomarkers could be also potential targets for therapeutic approach (38) which needs to be further explored in a proper clinical trial setting.

\section{Tumor treating fields (Optune)}

Tumor treating fields utilizes the natural electrical properties of dividing cancer cells and it exerts forces on highly polarized cellular proteins, tubulin and septin, preventing them from moving to their correct locations and disrupting cancer cell division and growth. This has shown some efficacy in the treatment of glioblastoma and, currently, there are two ongoing clinical trials using tumor treating fields alone or in combination with bevacizumab.

\section{Conclusions}

The treatment options for meningiomas have been very limited in the past with only surgery and radiation therapy as standard of care. Chemotherapy has been tried with modest results. Once the tumor recurs, the prognosis becomes poor. However, recent advances in diagnostics utilizing molecular genetic profiling, identification of specific genetic alterations and understanding its pathways and potential targets have opened up new therapeutic 
options in the management of meningiomas which are changing the paradigm of the clinical trial design that hopefully will, in return, improve the diagnostic and therapeutic outcome. A significant enthusiasm has been raised with the introduction of molecular targeted therapies and immunotherapies as our knowledge and understanding of vast complex tumor genomics continue to grow. If felt to be eligible, a participation in a well-designed clinical trial would be highly recommended.

\section{Acknowledgments}

Funding: None.

\section{Footnote}

Provenance and Peer Review: This article was commissioned by the Guest Editor (Jon Glass) for the series "Targeted systemic therapies for primary and metastatic brain tumors" published in Chinese Clinical Oncology. The article has undergone external peer review.

Reporting Checklist: The author has completed the Narrative Review reporting checklist. Available at http://dx.doi. org/10.21037/cco-2020-mbt-01

Conflicts of Interest: The author has completed the ICMJE uniform disclosure form (available at http://dx.doi. org/10.21037/cco-2020-mbt-01). The series "Targeted systemic therapies for primary and metastatic brain tumors" was commissioned by the editorial office without any funding or sponsorship. The author has no other conflicts of interest to declare.

Ethical Statement: The author is accountable for all aspects of the work in ensuring that questions related to the accuracy or integrity of any part of the work are appropriately investigated and resolved.

Open Access Statement: This is an Open Access article distributed in accordance with the Creative Commons Attribution-NonCommercial-NoDerivs 4.0 International License (CC BY-NC-ND 4.0), which permits the noncommercial replication and distribution of the article with the strict proviso that no changes or edits are made and the original work is properly cited (including links to both the formal publication through the relevant DOI and the license). See: https://creativecommons.org/licenses/by-nc-nd/4.0/.

\section{References}

1. Zhang AS, Ostrom QT, Kruchko C, et al. Complete prevalence of malignant primary brain tumors registry data in the United States compared with other common cancers, 2010. Neuro Oncol 2017;19:726-35.

2. Ostrom QT, Gittleman H, Xu J, et al. CBTRUS Statistical Report: Primary Brain and Other Central Nervous System Tumors Diagnosed in the United States in 2009-2013. Neuro Oncol 2016;18:v1-v75.

3. Plotkin SR, Blakeley JO, Evans DG, et al. Update from the 2011 International Schwannomatosis Workshop: From genetics to diagnostic criteria. Am J Med Genet Part A 2013;161A:405-16.

4. Rousseau G, Noguchi T, Bourdon V, et al. SMARCB1/ INI1 germline mutations contribute to $10 \%$ of sporadic schwannomatosis. BMC Neurol 2011;11:9.

5. Smith MJ, Isidor B, Beetz C, et al. Mutations in LZTR1 add to the complex heterogeneity of schwannomatosis. Neurology 2015;84:141-7.

6. Gerkes EH, Fock JM, den Dunnen WF, et al. A heritable form of SMARCE1-related meningiomas with important implications for follow-up and family screening. Neurogenetics 2016;17:83-9.

7. Al-Rashed M, Foshay K, Abedalthagafi M. Recent Advances in Meningioma Immunogenetics. Front Oncol 2020;9:1472.

8. Louis DN, Ohgaki H, Wiestler OD, et al. WHO Classification of Tumours of the Central Nervous System. WHO Classification of Tumours, 4th Edition, Volume 1. Lyon: IARC Press, 2016.

9. Clark VE, Erson-Omay EZ, Serin A et al. Genomic analysis of non-NF2 meningiomas reveals mutations in TRAF7, KLF4, AKT1, and SMO. Science 2013;339:1077-80.

10. Abedalthagafi M, Bi WL, Aizer AA et al. Oncogenic PI3K mutations are as common as AKT1 and SMO mutations in meningioma. Neuro Oncol 2016;18:649-55.

11. Abedalthagafi MS, Merrill PH, Bi WL et al. Angiomatous meningiomas have a distinct genetic profile with multiple chromosomal polysomies including polysomy of chromosome 5. Oncotarget 2014;5:10596-606.

12. Bi WL, Zhang $M, W u W W$, et al. Meningioma genomics: diagnostic, prognostic, and therapeutic applications. Front Surg 2016;3:40.

13. Ostrom QT, Cioffi G, Gittleman H, et al. CBTRUS Statistical Report: Primary Brain and Other Central Nervous System Tumors Diagnosed in the United States in 2012-2016. Neuro Oncol 2019;21:v1-v100. 
14. Chamberlain MC. Hydroxyurea for recurrent surgery and radiation refractory high-grade meningioma. J Neurooncol 2012;107:315-21.

15. Kim J, Kim KH, Kim YZ. The clinical outcome of hydroxyurea chemotherapy after incomplete resection of atypical meningiomas. Brain Tumor Res Treat 2017;5:77-86.

16. Chamberlain MC, Adjuvant combined modality therapy for malignant meningioma. J Neurosurg 1996:84:733-6

17. Chamberlain MC, Tsao-Wei DD, Groshen S. Temozolomide for treatment-resistant recurrent meningioma. Neurology 2004;62:1210-2.

18. Gupta S, Bi WL, Dunn IF. Medical management of meningioma in the era of precision medicine. Neurosurg Focus 2018;44:E3.

19. Simó M, Argyriou AA, Macià M, et al. Recurrent highgrade meningioma: a phase II trial with somatostatin analogue therapy. Cancer Chemother Pharmacol 2014;73:919-23.

20. Norden AD, Ligon KL, Hammond SN, et al. Phase II study of monthly pasireotide LAR (SOM230C) for recurrent or progressive meningioma. Neurology 2015;84:280-6.

21. Graillon T, Romano D, Defilles C, et al. Octreotide therapy in meningiomas: in vitro study, clinical correlation, and literature review. J Neurosurg 2017;127:660-9.

22. Norden AD, Raizer JJ, Abrey LE, et al. Phase II trials of erlotinib or gefitinib in patients with recurrent meningioma. J Neurooncol 2010;96:211-7.

23. Wen PY, Yung WK, Lamborn KR, et al. Phase II study of imatinib mesylate for recurrent meningiomas (North American Brain Tumor Consortium study 01-08). Neuro Oncol 2009;11:853-60.

24. Karajannis MA, Legault G, Hagiwara M, et al. Phase II study of everolimus in children and adults with neurofibromatosis type 2 and progressive vestibular schwannomas. Neuro Oncol 2014;16:292-7.

25. Kaley TJ, Wen P, Schiff D, et al. Phase II trial of sunitinib for recurrent and progressive atypical and anaplastic meningioma. Neuro Oncol 2015;17:116-21.

26. Grimm SA, Kumthekar P, Chamberlain MC, et al. Phase II trial of bevacizumab in patients with surgery and radiation refractory progressive meningioma. J Clin Oncol 2015;33:abstr 2055.

27. Shih KC, Chowdhary S, Rosenblatt P, et al. A phase II trial of bevacizumab and everolimus as treatment for patients with refractory, progressive intracranial meningioma. J Neurooncol 2016;129:281-8.
28. Grallion T, Sanson M, Peyre M, et al. A phase II of everolimus and octreotide for patients with refractory and documented progressive meningioma (CEVOREM). J Clin Oncol 2017;35:abstr 2011.

29. Weller M, Roth P, Sahm F, et al. Durable Control of Metastatic AKT1-Mutant WHO Grade 1 Meningothelial Meningioma by the AKT Inhibitor, AZD5363. J Natl Cancer Inst 2017;109:1-4.

30. Collord G, Tarpey P, Kurbatova N, et al. An integrated genomic analysis of anaplastic meningioma identifies prognostic molecular signatures. Sci Rep 2018;8:13537.

31. Shankar GM, Abedalthagafi M, Vaubel RA, et al. Germline and somatic BAP1 mutations in high-grade rhabdoid meningiomas. Neuro Oncol 2017;19:535-45.

32. Boström J, Meyer-Puttlitz B, Wolter M, et al. Alterations of the tumor suppressor genes CDKN2A (p16(INK4a)), p14(ARF), CDKN2B (p15(INK4b)), and CDKN2C (p18(INK4c)) in atypical and anaplastic meningiomas. Am J Pathol 2001;159:661-9

33. Goutagny S, Yang HW, Zucman-Rossi J, et al. Genomic profiling reveals alternative genetic pathways of meningioma malignant progression dependent on the underlying NF2 status. Clin Cancer Res 2010;16:4155-64.

34. Juratli TA, Thiede C, Koerner MVA, et al. Intratumoral heterogeneity and TERT promoter mutations in progressive/higher grade meningiomas. Oncotarget 2017;8:109228-37.

35. Chamberlain MC, Glantz MJ. Interferon-alpha for recurrent World Health Organization grade 1 intracranial meningiomas. Cancer 2008;113:2146-51.

36. Dunn IF, Du Z, Touat $M$, et al. Mismatch repair deficiency in high-grade meningioma: a rare but recurrent event associated with dramatic immune activation and clinical response to PD-1 blockade. JCO Precis Oncol 2018;2018:PO.18.00190.

37. Giles AJ, Hao S, Padget M, et al. Efficient ADCC killing of meningioma by avelumab and a high-affinity natural killer cell line, haNK. JCI Insight 2019;4:e130688.

38. Everson RG, Hashimoto Y, Freeman JL, et al. Multiplatform profiling of meningioma provides molecular insight and prioritization of drug targets for rational clinical trial design. J Neurooncol 2018;139:469-78.

Cite this article as: Kim L. A narrative review of targeted therapies in meningioma. Chin Clin Oncol 2020;9(6):76. doi: 10.21037/cco-2020-mbt-01 